\title{
Nocturnal heart rate variability in obstructive sleep apnoea: a cross-sectional analysis of the Sleep Heart Health Study
}

\author{
Matteo Bradicich ${ }^{1}$, Noriane A. Sievi ${ }^{1}$, Fabian A. Grewe ${ }^{1}$, Alessio Gasperetti ${ }^{2}$, Malcolm Kohler ${ }^{1,3}$, \\ Esther I. Schwarz ${ }^{1,3}$ \\ ${ }^{1}$ Department of Pulmonology and Sleep Disorders Centre, University Hospital Zurich, Zurich, Switzerland; ${ }^{2}$ University Heart Center, University \\ Hospital Zurich, Zurich, Switzerland; ${ }^{3}$ Center of Competence Sleep and Health, University of Zurich, Zurich, Switzerland \\ Contributions: (I) Conception and design: M Bradicich, EI Schwarz, FA Grewe; (II) Administrative support: EI Schwarz, M Kohler; (III) Provision \\ of study materials or patients: None; (IV) Collection and assembly of data: None; (V) Data analysis and interpretation: All authors; (VI) Manuscript \\ writing: All authors; (VII) Final approval of manuscript: All authors. \\ Correspondence to: Esther I. Schwarz, MD. Department of Pulmonology and Sleep Disorders Centre, University Hospital Zurich, Raemistrasse 100, \\ CH-8091, Zurich, Switzerland. Email: estherirene.schwarz@usz.ch.
}

Background: Obstructive sleep apnoea (OSA) results in sympathetic overdrive. Increased nocturnal heart rate variability (HRV) is a surrogate marker of autonomic disturbance. The aim was to study the association of the apnoea-hypopnea index (AHI), nocturnal hypoxaemia, and sleep fragmentation with nocturnal HRV to address the pathophysiological mechanisms underlying autonomic disturbance in OSA.

Methods: Participants of the Sleep Hearth Health Study with available data on nocturnal HRV and an AHI $\geq 10 / \mathrm{h}$ have been included in this cross-sectional analysis. The main outcome of interest was the association of sleep fragmentation, nocturnal hypoxaemia, and the AHI with nocturnal HRV. Multivariate regression modelling with the mean of the standard deviations of normal-sinus-to-normal-sinus-interbeat intervals in all 5-minute segments (SDNNIDX) and with low to high frequency power-ratio (LF/HF) as dependent variables controlling for prespecified confounders (age, sex, cups of coffee, beta blocker, nocturnal heart rate) was used to assess the contribution of the arousal index, total sleep time with an oxygen saturation $<90 \%$ (TST90) and the AHI not due to arousals to HRV. The significance level was set at $\mathrm{P}<0.01$.

Results: In 258 patients with OSA (mean \pm SD age $62 \pm 10$ years, BMI $29 \pm 4 \mathrm{~kg} / \mathrm{m}^{2}$, median (IQR) AHI 18.6/h (14.0-25.6), the arousal index (coef =0.42, $\mathrm{P}=0.002$ ) was independently positively associated with SDNNIDX also after having controlled for potential confounders, whereas the AHI (coef $=0.22$, $\mathrm{P}=0.030$ ) and TST90 (coef =0.36, $\mathrm{P}=0.054$ ) were not. The arousal index-but not TST and AHI-was also independently associated with $\mathrm{LF} / \mathrm{HF}$.

Conclusions: In OSA, pronounced sleep fragmentation is associated with higher nocturnal HRV and a sympatho-vagal imbalance with sympathetic dominance. OSA severity and nocturnal hypoxaemia did not independently predict nocturnal HRV.

Keywords: Obstructive sleep apnoea (OSA); heart rate variability (HRV); arousal index; intermittent hypoxia; sleep fragmentation

Submitted Jun 06, 2020. Accepted for publication Sep 05, 2020.

doi: $10.21037 /$ jtd-cus-2020-005

View this article at: http://dx.doi.org/10.21037/jtd-cus-2020-005

(c) Journal of Thoracic Disease. All rights reserved. 


\section{Introduction}

Obstructive sleep apnoea (OSA) defined by an elevated apnoea-hypopnoea-index (AHI) is highly prevalent in the adult population $(1,2)$. However, only a part of this OSA population has a clinically relevant OSA that indicates treatment based on symptoms, sleep quality, or cardiovascular comorbidities. There are different phenotypes with different symptom burden and different cardiovascular effects of OSA (3-5). There is a poor correlation between OSA severity based on the AHI and symptoms. However, in epidemiological studies, OSA severity was an independent predictor of cardiovascular consequences with a higher likelihood of hypertension and cardiovascular events in severe OSA in longitudinal studies (6-9). OSA is characterised by recurrent episodes of partial or complete upper airway obstruction during sleep, leading to nocturnal intermittent hypoxia, arousals, intrathoracic pressure oscillations, and hypercapnia, resulting in increased sympathetic activity and disturbed autonomic balance. Nocturnal intermittent hypoxia has been suggested as the most relevant underlying mechanism of sympathetic overdrive and elevated blood pressure in OSA models $(10,11)$. However, the role of sleep fragmentation in increasing sympathetic activity has been discussed as well (12-14).

The role of the AHI and the oxygen desaturation index (ODI) as conventional OSA severity markers in explaining cardiovascular effects of OSA is currently under discussion, probably due to their neglect of some crucial pathophysiologic factors that do not allow them to be used as marker of autonomic imbalance $(15,16)$. Autonomic disturbance induced by OSA might be an additional factor of prognostic relevance in OSA.

In order to assess the pathophysiologic interplay between OSA and sympathetic override, the autonomic nervous system has been investigated directly by means of microneurography as well as indirectly through baroreflex sensitivity, cold pressor test, handgrip test, measurement of urinary catecholamine excretion, and heart rate variability (HRV) analysis during sleep or wake (14). Nocturnal HRV is a surrogate marker of autonomic regulation and sympathovagal balance during sleep, and HRV has been shown to be altered in OSA. The prognostic role of nocturnal HRV regarding cardiovascular outcomes is currently under investigation (17). Moreover, it is acknowledged that stress is correlated with an increase in HRV during non-rapid eye movement (NREM) sleep, which represents the main part of sleep $(13,18)$. Among patients with OSA, a raise in HRV during sleep is probably related to the adrenergic bursts caused by intermittent hypoxia and arousals as well as intrathoracic pressure swings $(14,19)$. In addition, a decreased baroreflex control has been shown in OSA $(20,21)$, potentially also in association with hypertension. However, whether nocturnal intermittent hypoxia is independently associated with changes in HRV or whether it acts on sympathetic activation by triggering arousals is less clear.

In this cross-sectional analysis, sleep fragmentation and nocturnal hypoxia have been studied as potential HRV modulators in a real-life population of subjects with OSA in order to assess the role of the pathophysiological mechanisms involved in OSA on autonomic imbalance.

The main hypothesis of this study was that in OSA, both markers of sleep fragmentation and nocturnal hypoxaemia are associated with an increase in nocturnal HRV (Figure 1). We present the following article in accordance with the STROBE checklist (available at http://dx.doi.org/10.21037/ jtd-cus-2020-005).

\section{Methods}

\section{Data source}

The Sleep Heart Health Study (22) enrolled men and women aged 40 years and older to undergo a polysomnography (PSG) between 1995 and 1998. The data of the polysomnographic recording of these participants has been used to conduct HRV analysis $(n=486)$. These data were available for download from the website of the National Sleep Research Resource (https://sleepdata.org). The download and usage of the data has been authorised by The Brigham and Women's Hospital through its Division of Sleep and Circadian Disorders (Boston, MA, USA) after the submission and acceptance of a Data Access and Use Agreement containing the details of this crosssectional analysis. The National Sleep Research Resource offers free access to large collections of de-identified physiological signals and clinical data elements collected in well-characterised research cohorts and clinical trials and is supported by Grant Number HL114473 from the National Heart, Lung, and Blood Institute, National Institutes of Health (USA). The study was conducted in accordance with the Declaration of Helsinki and the Harmonized Tripartite Guideline for Good Clinical Practice from the International Conference on Harmonization. This study was reviewed and approved by the institutional review boards of each site 


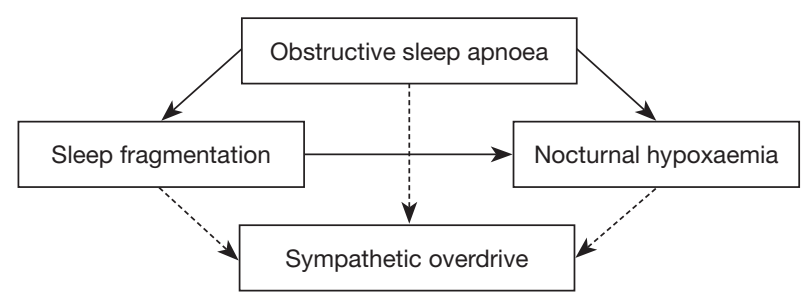

Figure 1 Interplay between sleep fragmentation, intermittent nocturnal desaturation, and sympathetic override in OSA. The dotted lines represent the investigated relationship between OSA, arousals, intermittent nocturnal desaturations, and sympathetic activation. OSA, obstructive sleep apnoea.

of the SHHS centres. All patients enrolled in the SHHS completed the informed consent form.

\section{Eligibility criteria}

Participants from the initial cohort have been excluded if they either presented with or reported atrial fibrillation or a higher degree atrioventricular block at the time of PSG, since these arrhythmias affect HRV analysis. Furthermore, only participants with a total sleep time of at least 240 minutes and OSA defined by an AHI $\geq 10$ episodes per hour of sleep have been included. The threshold of 10 events per hour has been chosen in order to exclude borderline AHI values that might not reflect OSA (23-28). AHI was defined as the number of all apnoeas and hypopnoeas associated with an oxygen desaturation of at least $3 \%$ or an arousal per hour of sleep (29). For the definitions of the measurement methods please refer to the original paper (22) or the study protocol available on the website of the National Sleep Research Resource. According to the eligibility criteria, data of 258 participants were available for the analysis (Figure 2).

\section{Extracted data}

The following data were extracted for this analysis: age, sex, ethnicity, BMI, neck circumference, presence of atrial fibrillation, hypertension, heart failure, types of antihypertensive drugs, diabetes mellitus, smoking, pack years, cups of coffee within four hours before the sleep study, apnoea-hypopnea-index ( $3 \%$ \& 4\% dips) with and without arousals, \% time with $\mathrm{SpO}_{2}<90 \%$, mean nocturnal $\mathrm{SpO}_{2}$, sleep stages, time in bed, sleep efficiency, arousal index, blood pressure, heart rate, measures of HRV (normal

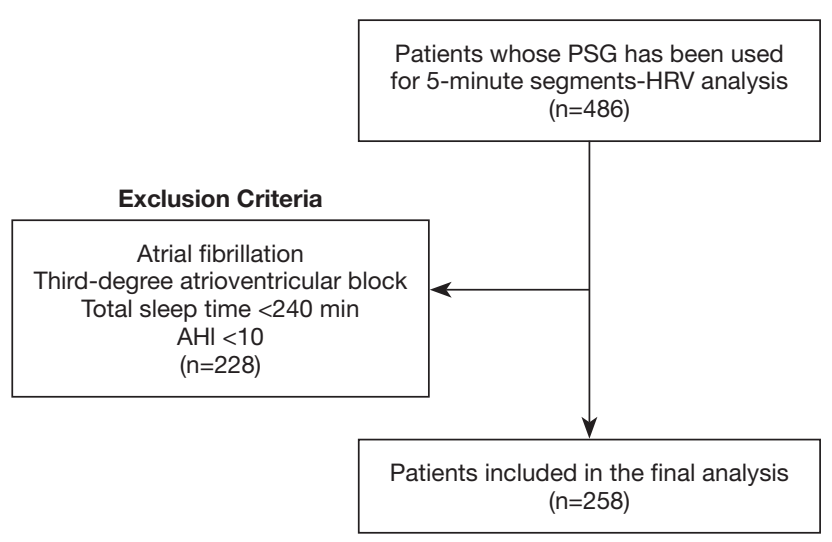

Figure 2 Patient flow. AHI, apnoea-hypopnea index; HRV, heart rate variability.

sinus-to-normal sinus interbeat intervals in all 5 -minute segments, ratio of low to high frequency power).

\section{Outcomes}

The main outcome of interest was the association of different pathophysiological features of OSA derived from PSG parameters-the arousal index, the percentage of the total sleep time with an oxygen saturation below $90 \%$ (TST90), and the AHI based on apnoeas and hypopnoeas associated with 3\%-oxygen desaturations and not on arousals-with nocturnal HRV assessed in the time domain by normal sinus-to-normal sinus interbeat $(\mathrm{NN})$ intervals in all 5-minute segments (SDNNIDX). This reflects mainly short-term HRV influenced by the autonomic nervous system.

A secondary outcome of interest was the association of these three PSG-derived parameters with nocturnal HRV in the time-frequency domain, quantified with the ratio of low to high frequency power (LF/HF) as marker of sympathetic to parasympathetic balance. The low and high frequency power reflect the $\mathrm{NN}$ interval spectral power between 0.04 and $0.15 \mathrm{~Hz}$ and between 0.15 and $0.4 \mathrm{~Hz}$, respectively. The HF is suggested to represent vagal modulation and is more affected by breathing (30).

\section{Statistical analysis}

In order to test the main hypothesis, we conducted a multiple regression analysis using SDNNIDX as dependent variable, expressed in ms. The HRV analysis of consecutive 5 minute-segments, whose results were downloaded from 
Table 1 Baseline characteristics of the analysed cohort

\begin{tabular}{|c|c|}
\hline Variable & Data of the cohort \\
\hline $\mathrm{N}$ & 258 \\
\hline Age, y & $62.3 \pm 9.8$ \\
\hline Female, n (\%) & $105[41]$ \\
\hline $\mathrm{BMI}, \mathrm{kg} / \mathrm{m}^{2}$ & $28.5 \pm 4.2$ \\
\hline Beta-blocker therapy, n (\%) & $12[5]$ \\
\hline Cups of coffee before bedtime, $n$ & $0.06 \pm 0.42$ \\
\hline Systolic office blood pressure, $\mathrm{mmHg}$ & $124.6 \pm 17.2$ \\
\hline Diastolic office blood pressure, $\mathrm{mmHg}$ & $73.8 \pm 10.0$ \\
\hline Arousal Index, n/h - median (IQR) & $19.0(13.9-25.5)$ \\
\hline TST90, \% - median (IQR) & $0.5(0.04-2.0)$ \\
\hline Total Sleep Time, min & $375 \pm 50$ \\
\hline AHla, $n / h$ - median (IQR) & $18.6(14.0-25.6)$ \\
\hline AHI, n/h - median (IQR) & $14.9(9.6-21.3)$ \\
\hline AHIa 10-14.9/h, n (\%) & $82(31.8)$ \\
\hline AHla 15-29.9/h, n (\%) & $130(50.4)$ \\
\hline AHla $>30 / h, n(\%)$ & $46(17.8)$ \\
\hline SDNNIDX, ms - median (IQR) & $52.0(40.8-65.6)$ \\
\hline LF/HR - median (IQR) & $2.2(1.3-3.4)$ \\
\hline
\end{tabular}

If not otherwise specified, data are shown as mean $\pm \mathrm{SD}$. AHI, apnoea-hypopnea index with hypopnoeas based on 3\%-oxygen desaturations only; AHIa, apnoea-hypopnoea index with hypopnoeas based on both 3\%-oxygen desaturations and arousals. IQR, interquartile range; SD, standard deviation; TST90, total sleep time with peripheral oxygen saturation below $90 \%$; SDNNIDX, mean of the standard deviations of normal sinus-to-normal sinus interbeat (NN) intervals in all 5-minute segments; LF/HF, low to high frequency power.

the website of the National Sleep Research Resource (https://sleepdata.org), has been conducted restricting the analysis to interbeat $(\mathrm{NN})$ intervals below 2.5 seconds, and to only 5 minute-windows with at least 150 normal sinus beats. There was no overlap between the analysed 5 -minute segments. To address the role of different aspects of OSA in nocturnal HRV, three different models with SDNNIDX (HRV) as dependent variable were created including either the arousal index as marker of sleep fragmentation, the TST90 as marker of nocturnal hypoxaemia, or the AHI not due to arousals (comparable to ODI as marker of intermittent hypoxia and conventional OSA severity) as independent variables, each model controlling for age, sex, use of beta-blockers, number of cups of coffee within 4 hours before bedtime, and heart rate. Since the ODI was not available in the dataset, the AHI based on $3 \%$-desaturations only and not on arousals was used as estimate of intermittent nocturnal hypoxaemia. The same regression analyses were performed using $\mathrm{LF} / \mathrm{HF}$ ratio as dependent variables.

Residual analysis of the models has been performed to check the regression assumption. The inclusion of the prespecified controlling variables has been motivated according to current literature and physiology evidence.

Descriptive statistics were reported across the patient sample, with means and standard deviation (SD) for continuous variables as well as numbers and percentages for categorical variables.

Considering an overall $\alpha$ significance level of 0.05 and four regression models, a two-sided $\mathrm{P}$ value below 0.01 has been considered statistically significant according to a Bonferroni correction.

Statistical analyses have been performed with Stata/SE 15.1 (TX, USA).

\section{Other relevant information}

The study has been conducted according to the Strengthening the reporting of observational studies in epidemiology (STROBE) Statement for cross-sectional studies (31).

\section{Results}

The cohort consisted of 258 middle-aged (mean \pm SD age of $62.3 \pm 9.8$ years) men $(59 \%)$ and women $(41 \%)$ with overweight (mean \pm SD BMI of $28.5 \pm 4.2 \mathrm{~kg} / \mathrm{m}^{2}$ ) with mostly mild to moderate obstructive sleep apnea $(\mathrm{AHI}>30 / \mathrm{h}$ in $18 \%$ ) without relevant hypoxic burden.

The baseline characteristics of the cohort are reported in Table 1.

The regression models studying the association of the arousal index as marker of sleep fragmentation, TST90 as marker of nocturnal hypoxaemia, and the AHI not based on arousals as marker of OSA severity and as surrogate marker of intermittent hypoxaemia (since ODI was not available) with nocturnal HRV (SDNNIDX) used age, sex, beta blocker therapy, cups of coffee within 4 hours before bedtime, and mean nocturnal heart rate as prespecified covariates. A statistically significant positive association between the arousal index and nocturnal HRV as dependent variable (SDNNIDX) was found also after having controlled 
Table 2 Multiple linear regression analysis between SDNNIDX and the arousal index

\begin{tabular}{lccc}
\hline SDNNIDX, ms & Regression coefficient & Confidence interval & P value \\
\hline Arousal Index, $\mathrm{n} / \mathrm{h}$ & 0.42 & 0.16 to 0.68 & 0.002 \\
Age, $\mathrm{y}$ & -0.28 & -0.53 to -0.02 & 0.036 \\
Female sex & -5.06 & -10.06 to -0.07 & 0.047 \\
Beta-blocker therapy & -7.78 & -18.82 to 3.25 & 0.166 \\
Cups of coffee before bedtime, $\mathrm{n}$ & 6.22 & 0.41 to 12.04 & 0.036 \\
Heart rate, bpm & -1.21 & -1.51 to -0.91 & $<0.001$ \\
\hline
\end{tabular}

Adjusted $R^{2}=0.29$. SDNNIDX, mean of the standard deviations of normal sinus-to-normal sinus interbeat (NN) intervals in all 5-minute segments.

Table 3 Multiple linear regression analysis between SDNNIDX and TST90

\begin{tabular}{lccc}
\hline SDNNIDX, ms & Regression coefficient & Confidence Interval & P value \\
\hline TST90, $\%$ & 0.40 & 0.04 to 0.76 & -0.58 to -0.06 \\
Age, y & -0.32 & -9.85 to 0.24 & 0.017 \\
Female sex & -4.81 & -20.28 to 1.92 \\
Beta-blocker therapy & -9.18 & -0.46 to 11.17 & 0.062 \\
Cups of coffee before bedtime, $\mathrm{n}$ & 5.36 & -1.57 to $-0.96<$ & 0.071 \\
Heart rate, $\mathrm{n} /$ min & -1.27 & -0.09 to 0.01 & 0.001 \\
Total sleep time, $\mathrm{h}$ & -0.04 & 0.080 &
\end{tabular}

Adjusted $R^{2}=0.27$. SDNNIDX, mean of the standard deviations of normal sinus-to-normal sinus interbeat (NN) intervals in all 5-minute segments; TST90, total sleep time with peripheral oxygen saturation below $90 \%$. In this model, total sleep time has been added as a controlling variable since TST90 is not a frequency index.

for the above listed confounding factors with a coefficient of 0.42 (95\% CI, 0.16-0.68), $\mathrm{P}=0.002$ (Table 2). Using a level of significance of $\mathrm{P}<0.01$, there was no evidence for a statistically significant association between TST90 [coef. 0.40 (95\% CI, 0.04-0.76), $\mathrm{P}=0.03$; see Table 3] nor between the AHI not due to arousals [coef. 0.22 (95\% CI, 0.02-0.41), $\mathrm{P}=0.03$; see Table 4] and nocturnal HRV (SDNNIDX) after controlling for confounders.

In a regression model including all three parameters of interest - the arousal index, TST90, and the AHI not due to arousals-controlling for age, sex, use of beta blockers, cups of coffee within four hours before bedtime, and heart rate, the arousal index was the only variable independently associated with nocturnal HRV (SDNNIDX) among the analysed dependent variables [coef. 0.37 (95\% CI, 0.080.65), $\mathrm{P}=0.01$; see Table 5].

The regression models using the LF/HF ratio as dependent variable and controlling for the same covariates as above, demonstrated a statistically significant independent association between the arousal index and the LF/HF ratio [coef. 0.03 (95\% CI, 0.01-0.06), $\mathrm{P}=0.004$ ]. There was no evidence for an independent association between TST90 [coef. -0.03 (95\% CI, -0.06-0.00), P=0.09] and the LF/HF ratio nor between the $\mathrm{AHI}$ not due to arousals and the $\mathrm{LF}$ / HF ratio [coef. -0.01 (95\% CI, -0.03-0.01), $\mathrm{P}=0.34$ ].

\section{Discussion}

In this cross-sectional analysis of a subpopulation of the Sleep Heart Health Study with OSA, the impact of sleep fragmentation, nocturnal hypoxaemia, and OSA severity on short-term nocturnal HRV has been studied. The arousal index was independently associated with an increased HRV in both the time domain (SDNNIDX) and the timefrequency domain (LF/HF ratio) as the best predictor of HRV out of different sleep-study derived parameters 
Table 4 Multiple linear regression analysis between SDNNIDX and the AHI not due to arousals

\begin{tabular}{lccc}
\hline SDNNIDX, ms & Regression coefficient & Confidence interval & P value \\
\hline AHI, $\mathrm{n} / \mathrm{h}$ & 0.22 & 0.02 to 0.41 & -0.52 to -0.11 \\
Age, $\mathrm{y}$ & -0.27 & -9.93 to 0.25 \\
Female sex & -4.84 & -20.91 to 1.40 & 0.041 \\
Beta-blocker therapy & -9.76 & -0.52 to 11.16 & 0.086 \\
Cups of coffee before bedtime, $\mathrm{n}$ & 5.32 & -1.50 to 0.90 & 0.074 \\
Heart rate, $\mathrm{n} / \mathrm{min}$ & -1.20 & $<0.001$ \\
\hline
\end{tabular}

Adjusted $\mathrm{R}^{2}=0.27$. AHI, apnoea-hypopnoea index with hypopnoeas based on 3\%-oxygen desaturations only (not on arousals). SDNNIDX, mean of the standard deviations of normal sinus-to-normal sinus interbeat (NN) intervals in all 5-minute segments.

Table 5 Multiple linear regression analysis between SDNNIDX and arousal index, TST90, and AHI not due to arousals

\begin{tabular}{lccc}
\hline SDNNIDX, ms & Regression coefficient & Confidence interval & P value \\
\hline Arousal index, $\mathrm{n} / \mathrm{h}$ & 0.37 & 0.08 to 0.65 & 0.011 \\
TST90, $\%$ & 0.30 & -0.10 to 0.70 & 0.141 \\
AHI, $\mathrm{n} / \mathrm{h}$ & 0.03 & -0.20 to 0.27 & 0.768 \\
Age, $\mathrm{y}$ & -0.31 & -0.57 to -0.05 & 0.018 \\
Female sex & -4.68 & -9.74 to 0.37 & 0.069 \\
Beta-blocker therapy & -8.66 & -19.75 to 2.43 & 0.125 \\
Cups of coffee before bedtime, $\mathrm{n}$ & 5.99 & 0.18 to 11.80 & -1.55 to -0.95 \\
Heart rate, $\mathrm{n} /$ min & -1.25 & 0.043
\end{tabular}

Adjusted $R^{2}=0.30$. SDNNIDX, mean of the standard deviations of normal sinus-to-normal sinus interbeat (NN) intervals in all 5-minute segments; TST90, total sleep time with peripheral oxygen saturation below 90\%; AHI, apnoea-hypopnoea index with hypopnoeas based on 3\%-oxygen desaturations only (not on arousals).

representing sleep fragmentation, nocturnal hypoxaemia, and OSA severity. This suggests that sleep fragmentation is associated with autonomic cardiac imbalance during sleep in patients with OSA. An increase of 1 arousal per hour during the night was associated with a proportional increase of $0.42 \mathrm{~ms}$ of SDNNIDX, an estimate of HRV. Considering a median of 19 arousals per hour in the analysed cohort, a median increase of $8 \mathrm{~ms}$ of SDNNIDX can be estimated over the night according to the regression model, which represents an increase of $15 \%$ in nocturnal SDNNIDX (median $52 \mathrm{~ms}$ ). Measures of nocturnal hypoxaemia and OSA severity (TST90 and AHI not based on arousals, respectively) were not independently associated with nocturnal HRV in this cohort including patients with mild, moderate, and severe OSA that, however, consisted overall mainly of mild and moderate OSA ( $>80 \%)$. Considering that a dose-response relationship between $\mathrm{AHI}$ and $\mathrm{HRV}$ has been observed $(14,32)$, our findings suggest that this might mainly be explained by respiratory events associated with arousals (33).

Nocturnal short-term HRV has been used as surrogate marker for autonomic cardiac regulation and sympathovagal balance during sleep $(17,34,35)$. The autonomic nervous system modulates cardiac function during sleep. Physiologically, there is a gradually increasing vagal dominance during NREM sleep stages 1-3 but a higher sympathetic activity during REM sleep (36,37). In OSA, there is a shift of the sympatho-vagal balance towards sympathetic predominance (38). However, HRV during sleep in OSA cannot be solely attributed to autonomic neural regulation. Blood pressure surges during obstructive respiratory events influence heart rate via the baroreflex and respiration via parasympathetic-mediated respiratory sinus arrest. Moreover, enhanced chemoreflexes and non-neural 
factors like intrathoracic pressure oscillations play a role as well (37). Overall, the pattern of HRV is altered in OSA, but different changes have been described.

When interpreting HRV, attention should be paid to the differences between 24-hour HRV and short-term HRV during sleep based on 5-minutes-intervals-they are not interchangeable. Autonomic cardiac control is regulated differently during wake and during different sleep stages. A decreased long-term HRV (24 h) and an increased LF/HF ratio are seen in hypertension and the latter has been described in OSA as well $(39,40)$. A decreased HRV over 24 hours has been suggested as negative prognostic marker in cardiovascular disease (41). However, nocturnal short-term HRV based on SDNN reflects another component of HRV and-across all sleep stages-has been described in most but not all studies as longer in OSA compared to patients not having OSA (42-45).

In our study, the association between sleep fragmentation and SDNNIDX and LF/HF ratio were positive, suggesting an increased overall nocturnal HRV and a change in nocturnal autonomic function towards sympathetic activity. However, SDNNIDX was relatively low with $52 \mathrm{~ms}$. Based on data from 24-hour-HRV analysis, it was suggested that an SDNN $<50 \mathrm{~ms}$ is to be considered unhealthy, while an SDNN >100 ms reflects healthy (41). However, population means for SDNN and LF/HF ratio have been described as $50 \pm 16$ and $2.8 \pm 2.6 \mathrm{~ms}$, respectively (46).

The finding that only arousal-based measures of OSA severity were independently associated with HRV but not measures of nocturnal hypoxaemia or intermittent hypoxaemia was not in line with the hypothesis of an independent association between intermittent hypoxaemia and HRV. Whether this finding is only applicable to this cohort of patients with mild to moderate OSA with a minor hypoxic burden or also to cohorts with more pronounced nocturnal hypoxaemia needs to be further studied. However, other data support that HRV is closer related to autonomic arousals than to intermittent hypoxaemia. Supplemental oxygen resulted in a significant reduction of a rise in morning blood pressure compared to sham using a CPAP therapy withdrawal model in OSA. However, attenuation of intermittent hypoxaemia resulted only in a neglectable (although statistically significant) reduction of the pulse rise index-a pulse oximetry-derived measure of HRV that is used as surrogate marker of autonomic arousals (47). The findings of this randomised controlled cross-over trial support that autonomic control of the cardiovascular system is affected by several different pathophysiological consequences and also supports that HRV might be closer related to arousal-mediated autonomic disturbance than to intermittent hypoxaemiamediated sympathetic overdrive (47).

This study has several limitations. The main limitation of this cross-sectional analysis is that the study population (derived from a general population sample with an AHI $\geq 10 / \mathrm{h}$, not a sleep clinic population) consists mainly of patients with mild-to-moderate OSA. An OSA population with more cases with severe OSA and a relevant hypoxic burden would be more suitable to study the association between the pathophysiological constraints of OSA and nocturnal HRV. Another limitation is that ODI was not available and that the $\mathrm{AHI}$ including only hypopnoeas based on 3\%-desaturations and not on arousals had to be used as surrogate. In addition, intrathoracic pressure changes, which presumably affect HRV, were not assessed-however, oesophageal pressure is not a standard clinical assessment in OSA. HRV data were only available in a subset of the study population of the Sleep Heart Health Study, thus limiting the sample size for this analysis to a few hundreds. These limitations affect generalisability to a typical OSA population. Moreover, the statistical analysis revealed some degree of heteroscedasticity in the regression models.

We summarise that based on our findings, sleep fragmentation seems to have a relevant pathophysiological effect on HRV among patients with OSA. Moreover, nocturnal hypoxaemia per se appears not to account for an increased HRV in this OSA population with, however, only a limited hypoxic burden. Nevertheless, intermittent hypoxaemia might affect HRV by triggering arousals. In line with our findings, arousal index was demonstrated to be stronger associated with sympathetic overactivity than the AHI in other cohort studies involving patients with OSA (48).

Considering the interplay between OSA and autonomic cardiac dysfunction, the integration of HRV analysis in the diagnostic work-up of OSA might help to understand the role of sleep disordered breathing in autonomic dysregulation and support treatment recommendations for OSA. It has been shown that treatment of OSA by CPAP or hypoglossal nerve stimulation reduces the elevated HRV during sleep and that effective treatment of OSA reduces sympathetic override $(49,50)$. The impact of this reduction on vascular outcomes in OSA remains to be further analysed.

\section{Conclusions}

Among patients with OSA, sleep fragmentation is associated 
with an increased HRV and a shift of the sympatho-vagal balance to sympathetic dominance. This is important since it is well known that sympathetic overactivity results in increases in nocturnal and daytime blood pressure, one of the most important predictors of long-term cardiovascular outcomes.

\section{Acknowledgments}

Funding: None.

\section{Footnote}

Provenance and Peer Review: This article was commissioned by the Guest Editor (Joerg Steier) for the series " 5 th Clinical Update Sleep" published in Fournal of Thoracic Disease. The article was sent for external peer review.

Reporting Checklist: The authors have completed the STROBE reporting checklist. Available at http://dx.doi. org/10.21037/jtd-cus-2020-005

Data Sharing Statement: Available at http://dx.doi. org/10.21037/jtd-cus-2020-005

Conflicts of Interest: All authors have completed the ICMJE uniform disclosure form (available at http://dx.doi. org/10.21037/jtd-cus-2020-005). The series " $5^{\text {th }}$ Clinical Update Sleep" was commissioned by the editorial office without any funding or sponsorship. MK reports consulting fees from Bayer AG outside the submitted work. The authors have no other conflicts of interest to declare.

Ethical Statement: The authors are accountable for all aspects of the work in ensuring that questions related to the accuracy or integrity of any part of the work are appropriately investigated and resolved. The trial was conducted in accordance with the Declaration of Helsinki (as revised in 2013) and the Harmonized Tripartite Guideline for Good Clinical Practice from the International Conference on Harmonization. This study was reviewed and approved by the institutional review boards of each site of the SHHS centres. All patients enrolled in the SHHS completed the informed consent form.

Open Access Statement: This is an Open Access article distributed in accordance with the Creative Commons Attribution-NonCommercial-NoDerivs 4.0 International
License (CC BY-NC-ND 4.0), which permits the noncommercial replication and distribution of the article with the strict proviso that no changes or edits are made and the original work is properly cited (including links to both the formal publication through the relevant DOI and the license). See: https://creativecommons.org/licenses/by-nc-nd/4.0/.

\section{References}

1. Heinzer R, Vat S, Marques-Vidal P, et al. Prevalence of sleep-disordered breathing in the general population: the HypnoLaus study. Lancet Respir Med 2015;3:310-8.

2. Peppard PE, Young T, Barnet JH, et al. Increased prevalence of sleep-disordered breathing in adults. Am J Epidemiol 2013;177:1006-14.

3. Schwarz EI. Cardiovascular consequences of obstructive sleep apnea in different study models and novel perspectives. Curr Opin Pulm Med 2019;25:614-22.

4. Zinchuk AV, Jeon S, Koo BB, et al. Polysomnographic phenotypes and their cardiovascular implications in obstructive sleep apnoea. Thorax 2018;73:472-80.

5. Randerath W, Bassetti CL, Bonsignore MR, et al. Challenges and perspectives in obstructive sleep apnoea: Report by an ad hoc working group of the Sleep Disordered Breathing Group of the European Respiratory Society and the European Sleep Research Society. Eur Respir J 2018;52:1702616.

6. Kingshott RN, Sime PJ, Engleman HM, et al. Self assessment of daytime sleepiness: patient versus partner. Thorax 1995;50:994-5.

7. Nieto FJ, Young TB, Lind BK, et al. Association of sleepdisordered breathing, sleep apnea, and hypertension in a large community-based study. Sleep Heart Health Study. JAMA 2000;283:1829-36.

8. Young T, Finn L, Peppard PE, et al. Sleep disordered breathing and mortality: eighteen-year follow-up of the Wisconsin sleep cohort. Sleep 2008;31:1071-8.

9. Marin JM, Carrizo SJ, Vicente E, et al. Long-term cardiovascular outcomes in men with obstructive sleep apnoea-hypopnoea with or without treatment with continuous positive airway pressure: an observational study. Lancet 2005;365:1046-53.

10. Fletcher EC, Lesske J, Behm R, et al. Carotid chemoreceptors, systemic blood pressure, and chronic episodic hypoxia mimicking sleep apnea. J Appl Physiol (1985) 1992;72:1978-84.

11. Fletcher EC, Lesske J, Culman J, et al. Sympathetic denervation blocks blood pressure elevation in episodic 
hypoxia. Hypertension 1992;20:612-9.

12. Guo X, Zheng L, Wang J, et al. Epidemiological evidence for the link between sleep duration and high blood pressure: a systematic review and meta-analysis. Sleep Med 2013;14:324-32.

13. Seravalle G, Mancia G, Grassi G. Sympathetic Nervous System, Sleep, and Hypertension. Curr Hypertens Rep 2018;20:74.

14. Lombardi C, Pengo MF, Parati G. Obstructive sleep apnea syndrome and autonomic dysfunction. Auton Neurosci 2019;221:102563.

15. Azarbarzin A, Sands SA, Stone KL, et al. The hypoxic burden of sleep apnoea predicts cardiovascular diseaserelated mortality: the Osteoporotic Fractures in Men Study and the Sleep Heart Health Study. Eur Heart J 2019;40:1149-57.

16. Chen F, Chen K, Zhang C, et al. Evaluating the clinical value of the hypoxia burden index in patients with obstructive sleep apnea. Postgrad Med 2018;130:436-41.

17. Zhang L, Wu H, Zhang X, et al. Sleep heart rate variability assists the automatic prediction of long-term cardiovascular outcomes. Sleep Med 2020;67:217-24.

18. Hall M, Vasko R, Buysse D, et al. Acute stress affects heart rate variability during sleep. Psychosom Med 2004;66:56-62.

19. Coccagna G, Mantovani M, Brignani F, et al. Continuous recording of the pulmonary and systemic arterial pressure during sleep in syndromes of hypersomnia with periodic breathing. Bull Physiopathol Respir (Nancy) 1972;8:1159-72.

20. Noda A, Nakata S, Koike Y, et al. Continuous positive airway pressure improves daytime baroreflex sensitivity and nitric oxide production in patients with moderate to severe obstructive sleep apnea syndrome. Hypertens Res 2007;30:669-76.

21. Carlson JT, Hedner JA, Sellgren J, et al. Depressed baroreflex sensitivity in patients with obstructive sleep apnea. Am J Respir Crit Care Med 1996;154:1490-6.

22. Quan SF, Howard BV, Iber C, et al. The Sleep Heart Health Study: design, rationale, and methods. Sleep 1997;20:1077-85.

23. Kapur VK, Auckley DH, Chowdhuri S, et al. Clinical Practice Guideline for Diagnostic Testing for Adult Obstructive Sleep Apnea: An American Academy of Sleep Medicine Clinical Practice Guideline. J Clin Sleep Med 2017;13:479-504.

24. Peter JH, Fuchs E, Kohler U, et al. Studies in the prevalence of sleep apnea activity (SAA): evaluation of ambulatory screening results. Eur J Respir Dis Suppl 1986;146:451-8.

25. Le Bon O, Hoffman G, Tecco J, et al. Mild to moderate sleep respiratory events - One negative night may not be enough. Chest 2000;118:353-9.

26. Laing U, Fichter J. Screening measurements for sleep apnea: is a single measurement enough? Pneumologie 2002;56:673-8.

27. Stoberl AS, Schwarz EI, Haile SR, et al. Night-tonight variability of obstructive sleep apnea. J Sleep Res 2017;26:782-8.

28. Bittencourt LR, Suchecki D, Tufik S, et al. The variability of the apnoea-hypopnoea index. J Sleep Res 2001;10:245-51.

29. Berry RB, Budhiraja R, Gottlieb DJ, et al. Rules for scoring respiratory events in sleep: update of the 2007 AASM Manual for the Scoring of Sleep and Associated Events. Deliberations of the Sleep Apnea Definitions Task Force of the American Academy of Sleep Medicine. J Clin Sleep Med 2012;8:597-619.

30. Shaffer F, Ginsberg JP. An Overview of Heart Rate Variability Metrics and Norms. Front Public Health 2017;5:258.

31. von Elm E, Altman DG, Egger M, et al. The Strengthening the Reporting of Observational Studies in Epidemiology (STROBE) statement: guidelines for reporting observational studies. Epidemiology 2007;18:800-4.

32. Park DH, Shin CJ, Hong SC, et al. Correlation between the severity of obstructive sleep apnea and heart rate variability indices. J Korean Med Sci 2008;23:226-31.

33. Berry RB, Brooks R, Gamaldo C, et al. AASM Scoring Manual Updates for 2017 (Version 2.4). J Clin Sleep Med 2017;13:665-6.

34. Stein $\mathrm{PK}, \mathrm{Pu} \mathrm{Y}$. Heart rate variability, sleep and sleep disorders. Sleep Med Rev 2012;16:47-66.

35. Dingli K, Assimakopoulos T, Wraith PK, et al. Spectral oscillations of RR intervals in sleep apnoea/hypopnoea syndrome patients. Eur Respir J 2003;22:943-50.

36. Somers VK, Dyken ME, Mark AL, et al. Sympatheticnerve activity during sleep in normal subjects. N Engl J Med 1993;328:303-7.

37. Narkiewicz K, Somers VK. Cardiovascular variability characteristics in obstructive sleep apnea. Auton Neurosci 2001;90:89-94.

38. Narkiewicz K, Pesek CA, Kato M, et al. Baroreflex control of sympathetic nerve activity and heart rate in obstructive sleep apnea. Hypertension 1998;32:1039-43. 
39. Aeschbacher S, Bossard M, Schoen T, et al. Heart Rate Variability and Sleep-Related Breathing Disorders in the General Population. Am J Cardiol 2016;118:912-7.

40. Palma JA, Iriarte J, Fernandez S, et al. Long-term continuous positive airway pressure therapy improves cardiac autonomic tone during sleep in patients with obstructive sleep apnea. Clin Auton Res 2015;25:225-32.

41. Kleiger RE, Miller JP, Bigger JT Jr, et al. Decreased heart rate variability and its association with increased mortality after acute myocardial infarction. Am J Cardiol 1987;59:256-62.

42. Yang A, Schafer H, Manka R, et al. Influence of obstructive sleep apnea on heart rate turbulence. Basic Res Cardiol 2005;100:439-45.

43. Kim YS, Kim SY, Park DY, et al. Clinical Implication of Heart Rate Variability in Obstructive Sleep Apnea Syndrome Patients. J Craniofac Surg 2015;26:1592-5.

44. Zhu K, Chemla D, Roisman G, et al. Overnight heart rate variability in patients with obstructive sleep apnoea: a time and frequency domain study. Clin Exp Pharmacol Physiol 2012;39:901-8.

45. Roche F, Gaspoz JM, Court-Fortune I, et al. Screening of obstructive sleep apnea syndrome by heart rate variability analysis. Circulation 1999;100:1411-5.

46. Nunan D, Sandercock GR, Brodie DA. A quantitative systematic review of normal values for short-term heart rate variability in healthy adults. Pacing Clin Electrophysiol 2010;33:1407-17.

47. Turnbull CD, Sen D, Kohler M, et al. Effect of Supplemental Oxygen on Blood Pressure in Obstructive Sleep Apnea (SOX). A Randomized Continuous Positive Airway Pressure Withdrawal Trial. Am J Respir Crit Care Med 2019;199:211-9.

48. Kim JB, Seo BS, Kim JH. Effect of arousal on sympathetic overactivity in patients with obstructive sleep apnea. Sleep Med 2019;62:86-91.

49. Guo W, Lv T, She F, et al. The impact of continuous positive airway pressure on heart rate variability in obstructive sleep apnea patients during sleep: A metaanalysis. Heart Lung 2018;47:516-24.

50. Dedhia RC, Shah AJ, Bliwise DL, et al. Hypoglossal Nerve Stimulation and Heart Rate Variability: Analysis of STAR Trial Responders. Otolaryngol Head Neck Surg 2019;160:165-71.
Cite this article as: Bradicich M, Sievi NA, Grewe FA, Gasperetti A, Kohler M, Schwarz EI. Nocturnal heart rate variability in obstructive sleep apnoea: a cross-sectional analysis of the Sleep Heart Health Study. J Thorac Dis 2020;12(Suppl 2):S129-S138. doi: 10.21037/jtd-cus-2020-005 